\title{
Introduction. Vulnérabilités et incidences sur les parcours d'entrée dans la vie adulte : les jeunes une population spécifique?
}

Introduction. Vulnerabilities and impacts on the pathways to adulthood: young people a specific population?

Yaëlle Amsellem-Mainguy et Philippe Cordazzo

\section{(C) OpenEdition}

\section{Édition électronique}

URL : https://journals.openedition.org/popvuln/983

DOI : 10.4000/popvuln.983

ISSN : 2650-7684

Éditeur

LIR3S - Laboratoire Interdisciplinaire de Recherche "Sociétés Sensibilités Soin" (UMR 7366 CNRS-uB)

\section{Édition imprimée}

Date de publication : 1 juin 2018

Pagination : 9-17

ISBN : 978-2-918173-23-6

ISSN : 2269-0182

\section{Référence électronique}

Yaëlle Amsellem-Mainguy et Philippe Cordazzo, «Introduction. Vulnérabilités et incidences sur les parcours d'entrée dans la vie adulte : les jeunes une population spécifique? », Populations vulnérables [En ligne], 4 | 2018, mis en ligne le 12 juillet 2019, consulté le 26 février 2022. URL : http:// journals.openedition.org/popvuln/983 ; DOI : https://doi.org/10.4000/popvuln.983

Ce document a été généré automatiquement le 26 février 2022.

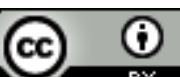

Les contenus de la revue Populations vulnérables sont mis à disposition selon les termes de la Licence Creative Commons Attribution 4.0 International 


\section{Introduction. Vulnérabilités et incidences sur les parcours d'entrée dans la vie adulte : les jeunes une population spécifique?}

Introduction. Vulnerabilities and impacts on the pathways to adulthood: young people a specific population?

Yaëlle Amsellem-Mainguy et Philippe Cordazzo

1 Au $1^{\text {er }}$ janvier 2018, la France compte 67,2 millions d'habitants. Le vieillissement de la population française se poursuit. Les personnes de 65 ans ou plus représentent 19,6\% de la population, contre $19,2 \%$ un an auparavant. Vingt ans plus tôt, elles ne représentaient que $15,5 \%$ de la population. À l'opposé, la population âgée de moins de 20 ans représente $24,4 \%$ de la population (contre $26 \%$ en 1998) (Papon et Beaumel, 2018). Parmi les jeunes, les données Eurostat montrent que 9,3\% d'entre eux sortent de manière précoce du système scolaire, c'est-à-dire sans diplôme, même si une partie a continué au lycée sans obtenir le baccalauréat. Dans un contexte où l'élévation du niveau de qualification de la population et la diminution de la part des peu diplômés sont nettes, les difficultés pour les jeunes non diplômés sont particulièrement fortes dans un pays où l'on sait l'importance des liens entre diplôme et insertion professionnelle (Gaubert et al., 2017).

2 À ces données de contexte, on peut ajouter que les jeunes adultes (20 à 29 ans) représentent $17 \%$ des personnes pauvres (INSEE, 2014). Il s'agit souvent de jeunes peu qualifiés, en difficulté d'insertion sur le marché du travail, au chômage et mal indemnisés.

3 Ces données invitent donc à la prudence lorsqu'on parle de «jeunesse », comme le soulignait Bourdieu, «la jeunesse n'est qu'un mot», avant tout pour souligner qu'il était illusoire de rassembler sous un même terme des expériences aussi diverses que celle de la «jeunesse dorée » et celle de la jeunesse populaire (1978). L'acception par la 
dimension générationnelle a en effet tendance à lisser la diversité des réalités socioéconomiques et inégalités traversant cette population dont les bornes d'âges fluctuent d'une enquête à une autre. Car bien qu'il soit difficile définir au sens strict la jeunesse, autrement que dans une perspective des âges de la vie (Van de Velde, 2016), il n'est aujourd'hui plus à démontrer la dimension cumulative des inégalités : origine sociale, ethno-raciale, sexe, diplôme, territoire mais aussi espace de vie (Labadie, 2014). S'il est intéressant de croiser cet âge de la vie qu'est la jeunesse avec la question des vulnérabilités, c'est bien que l'on a tendance à considérer dans le sens commun que les jeunes seraient plus vulnérables que les autres générations. Pour comprendre les enjeux du débat, il convient de revenir sur la notion même de vulnérabilité.

4 Tout d'abord les travaux en sociologie montrent que la vulnérabilité est a priori universelle (Soulet, 2014), dans la mesure où nous sommes tous vulnérables (même si individuellement nous ne sommes pas tous concernés de la même façon), elle est aussi potentielle (avec la concrétisation éventuelle d'un risque), relationnelle et contextuelle (c'est-à-dire relative à un contexte donné), enfin elle est réversible (on considère en effet qu'il s'agit davantage de "situations de vulnérabilités", pour souligner le caractère temporaire et la "possibilité » d'agir sur les facteurs et le contexte). Autrement dit, si tous les individus sont considérés a priori comme vulnérables potentiellement, l'exposition à des situations de vulnérabilité n'est pas la même pour tous. Lorsque l'on s'intéresse à la période de la jeunesse, ces constats prennent tout leur sens. Car les jeunes sont vulnérables au même titre que le reste de la population.

Néanmoins, ils se construisent en tant qu'individus et s'intègrent dans la société dans un contexte d'incertitude où leur vécu et leur parcours sont le résultat de l'articulation entre leurs ressources sociales et leur capacité à s'adapter aux normes de la société et à accepter individuellement les conséquences de leurs choix. La question de la vulnérabilité se poserait à ce niveau-là. Le non-accès et la non-possession des attributs, qu'ils soient matériels ou relationnels, ou la difficulté à se construire comme jeune, induiraient une situation de vulnérabilité (Becquet, 2012). Les analyses des conséquences de l'allongement de la jeunesse, bien qu'elles ne s'y réfèrent pas directement, pointent en fait des états, des situations ou des processus de vulnérabilité qui semblent s'accentuer actuellement, mais qui sont aussi considérés comme inhérents à cet âge de la vie. C'est ce que montre par exemple l'enquête sur les ressources des jeunes (ENRJ, Insee) qui pointe que l'aide financière des parents accompagne souvent la vie des jeunes adultes, au moment de leur transition vers l'indépendance (plus des deux tiers d'entre eux $-70 \%$ - reçoivent une aide financière régulière de leurs parents sous forme d'un versement monétaire ou d'une participation à leurs dépenses). Cette enquête montre que tous les jeunes ne sont pas aidés dans les mêmes proportions : dans tous les milieux sociaux ce sont les jeunes en cours d'études qui sont le plus souvent aidés et qui reçoivent l'aide la plus importante ; à l'opposé, chez les jeunes sortis du système éducatif, qu'ils occupent un emploi ou non, le soutien financier des parents est plus rare et moins élevé (Castell, Portela et Rivalin, 2016) (Tableau 1). Mais l'exposition plus forte des jeunes à des situations de vulnérabilités s'explique aussi et peut être avant tout par le fait que l'emploi des jeunes réagit plus vite et plus fortement aux variations du rythme de croissance de l'économie que l'emploi des adultes (Lefresne, 2003 ; Amsellem-Mainguy et Timoteo, 2012) comme les courbes du chômage le montrent bien (Figure 1). 
Tableau 1. Les aides financières régulières des parents aux jeunes adultes

\begin{tabular}{|c|c|c|c|c|c|c|c|c|c|}
\hline & \multirow{2}{*}{\begin{tabular}{|l} 
Reçoit \\
une aide \\
financière \\
régulière \\
(en \%)
\end{tabular}} & \multicolumn{7}{|l|}{ Dont : } & \multirow{2}{*}{$\begin{array}{ll}\text { Montant } & \\
\text { mensuel } & \\
\text { total } & \mathrm{d} \\
\text { l'aide } & \text { reçue } \\
\text { par } & \text { les } \\
\text { bénéficiaires } & \text { (en euros) }\end{array}$} \\
\hline & & $\begin{array}{l}\text { versement } \\
\text { monétaire }\end{array}$ & $\begin{array}{l}\text { aide } \\
\text { au } \\
\text { loyer }\end{array}$ & $\begin{array}{l}\text { aide } \\
\text { alimentaire }\end{array}$ & $\begin{array}{l}\text { aide aux } \\
\text { frais de } \\
\text { transports }\end{array}$ & $\begin{array}{l}\text { aide aux frais de } \\
\text { communications }\end{array}$ & $\begin{array}{l}\text { aide aux } \\
\text { dépenses } \\
\text { de loisirs }\end{array}$ & $\begin{array}{l}\text { aide à la } \\
\text { complémentaire } \\
\text { santé }\end{array}$ & \\
\hline \begin{tabular}{|l} 
Ensemble \\
des jeunes \\
adultes
\end{tabular} & 70 & 37 & 12 & 11 & 36 & 51 & 22 & 11 & 250 \\
\hline $\begin{array}{l}\text { En cours } \\
\text { d'études }\end{array}$ & 90 & 60 & 23 & 18 & 53 & 67 & 29 & 18 & 330 \\
\hline En emploi & 42 & 9 & 1 & 6 & 15 & 25 & 11 & 4 & 110 \\
\hline $\begin{array}{l}\text { Au } \\
\text { chômage } \\
\text { ou inactif* }\end{array}$ & 66 & 25 & 1 & 3 & 26 & 50 & 21 & 6 & 130 \\
\hline Cohabitants & 74 & 34 & $/ / /$ & $/ / /$ & 41 & 59 & 28 & 9 & 130 \\
\hline $\begin{array}{l}\text { En cours } \\
\text { d'études }\end{array}$ & 92 & 55 & $/ / /$ & $/ / /$ & 63 & 76 & 38 & 14 & 160 \\
\hline En emploi & 49 & 10 & $/ / /$ & $/ / /$ & 19 & 34 & 14 & 4 & 90 \\
\hline $\begin{array}{l}\mathrm{Au} \\
\text { chômage } \\
\text { ou inactif* }\end{array}$ & 74 & 28 & $/ / /$ & $/ / /$ & 31 & 60 & 26 & 7 & 120 \\
\hline $\begin{array}{l}\text { Semi- } \\
\text { cohabitants }\end{array}$ & 92 & 62 & 49 & 46 & 48 & 66 & 24 & 22 & 480 \\
\hline $\begin{array}{l}\text { En cours } \\
\text { d'études }\end{array}$ & 97 & 70 & 57 & 48 & 52 & 72 & 26 & 25 & 510 \\
\hline En emploi & 59 & 13 & 7 & 36 & 20 & 25 & 17 & 5 & 180 \\
\hline $\begin{array}{l}\text { Au } \\
\text { chômage } \\
\text { ou inactif* }\end{array}$ & ns & ns & ns & ns & ns & ns & $n s$ & ns & ns \\
\hline $\begin{array}{l}\text { Non- } \\
\text { cohabitants }\end{array}$ & 44 & 25 & 11 & 12 & 15 & 19 & 6 & 7 & 370 \\
\hline $\begin{array}{l}\text { En cours } \\
\text { d'études }\end{array}$ & 72 & 54 & 26 & 16 & 28 & 34 & 9 & 15 & 510 \\
\hline En emploi & 26 & 7 & 2 & 9 & 7 & 9 & 4 & 3 & 150 \\
\hline
\end{tabular}




\begin{tabular}{|l|l|l|l|l|l|l|l|l|l|}
\hline $\begin{array}{l}\text { Au } \\
\text { chômage } \\
\text { ou inactif* }\end{array}$ & 33 & 14 & 4 & 11 & 9 & 17 & 5 & 1 & 210 \\
\hline
\end{tabular}

* : hors études; ns : non significatif (effectifs insuffisants); /// : non concernés

Lecture : 70 \% des jeunes reçoivent au moins une aide financière de façon régulière ; $37 \%$ sont aidés sous la forme d'un versement monétaire, etc. Un jeune aidé reçoit en moyenne 250 euros par mois pour l'ensemble des aides financières régulières

Champ : personnes âgées de 18 à 24 ans résidant en France (hors Mayotte)

Source : DREES-INSEE, enquête nationale sur les ressources des jeunes 2014

Figure 1. Taux de chômage selon l'âge en 2016
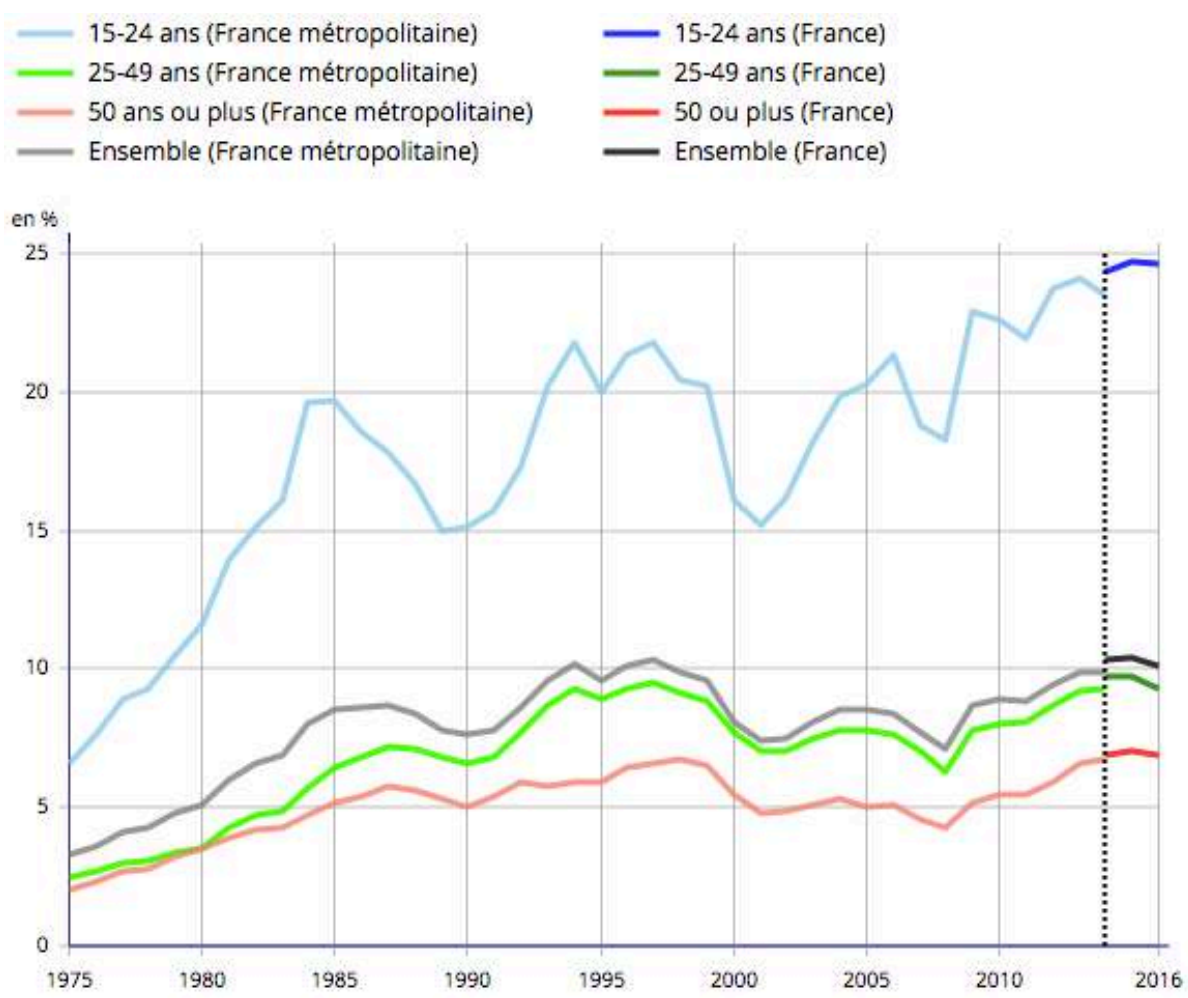

Lecture : en 2016, 24,6 \% des personnes âgées de 15 à 24 ans sont au chômage

Champ : France métropolitaine jusqu'en 2014 et France hors Mayotte à partir de 2014, population des ménages, personnes actives de 15 ans ou plus

Source : INSEE, enquêtes Emploi

Les jeunes sont dans une position particulière : ils sont à la fois vulnérables par rapport au chômage (leur probabilité d'entrer en chômage sur une période donnée est plus forte qu'à d'autres périodes de la vie) mais ils sont également plus «employables" (c'est-à-dire plus prompts à trouver un emploi au cours de la même période que les autres générations, bien que les conditions d'emploi et de travail ne soient pas toujours identiques, loin de là). Cela s'explique avant tout par leur âge : de fait ils ont pour la grande majorité d'entre eux une faible ancienneté sur le marché du travail, ce qui les place dans des positions de vulnérabilités plus fortes que le reste des actifs. Ils sont d'ailleurs plus touchés par des emplois précaires, mal rémunérés et qui peuvent être éloignés de leur domicile. Dès lors, dans les premiers temps de leur vie active, les jeunes se trouvent à accepter tous types d'emplois "faute de mieux», «en attendant». Ce 
constat largement partagé a donné l'occasion d'établir des dispositifs spécifiques, dédiés aux jeunes, dans l'objectif de les aider/accompagner à s'insérer dans la vie professionnelle.

Ce sont ces mêmes dispositifs qui auront pour effet de limiter parfois l'accès aux mêmes droits que ceux des autres salariés, «mais de telles mesures sont à double tranchant. Elles maintiennent le jeune dans un statut à part en fonction de son âge. Elles favorisent des présomptions sur les vulnérabilités du jeune, source de préjugés, et peuvent pérenniser aussi une précarisation discriminatoire de son statut. » (MercatBruns et Castany, 2016).

8 Les chercheur'e's ayant travaillé sur la question des vulnérabilités mettent en évidence que ce terme de "vulnérabilité » n'a rien d'une catégorie indigène (il n'est quasiment jamais employé par les « vulnérables » eux-mêmes, encore moins lorsqu'ils sont jeunes, issus des milieux les plus précaires) ne vaut que par l'analyse que portent de façon allogène non les acteurs de, mais les acteurs sur (Brodiez-Dolino, 2016). Cela invite donc à de grandes précautions dans l'usage et les catégories que l'on pourrait construire à partir de la "vulnérabilité", dans un contexte où les individus seraient tous potentiellement vulnérables, qui pointe en creux que ceux n'ayant pas les ressources pour s'adapter aux règles du jeu et répondre aux injonctions sociales le seraient encore davantage et paraîtraient à tort responsables de leur échec.

9 Afin de mieux comprendre ce qui est sous-jacent à la notion de «jeunes vulnérables », les cinq articles qui composent ce numéro abordent le phénomène dans une optique longitudinale. Ainsi, c'est bien l'inscription des «vulnérabilités » dans les parcours de vie de jeunes adultes ou en devenir qui est questionnée ici à partir d'un panel de parcours des plus originaux. Enfants d'immigrés, issus de la protection de l'enfance, incarcérés, bénéficiaires du RSA ou sortants de l'enseignement supérieur, tous traversent une période de leur vie de jeunes adultes associée à une vulnérabilité liée à leur statut et/ou leur parcours. Ainsi, si les travaux s'intéressent notamment à des populations dont le statut social renvoie à une notion de "vulnérabilité ", mais pas que (sortants du supérieur), l'ensemble des contributions mettent en évidence que tous les parcours peuvent être marqués par des situations de vulnérabilités. En effet, si le diplôme, comme nous l'avons vu précédemment, protège toujours les jeunes dans leurs parcours d'entrée dans la vie active, "signe de l'extension des phénomènes de déclassement, le diplôme ne protège pas systématiquement de la précarité » (Cordazzo et Sembel, 2016). À partir d'enquêtes et de méthodologies diverses, ce dossier a donc pour objectif de présenter des travaux récents sur la jeunesse qui, chacun à leur manière, interrogent la question des vulnérabilités.

10 L'article d'Isabelle Fréchon et Lucy Marquet ouvre le dossier en proposant de partir des enjeux de la prise en charge par le contrat jeune majeur (CJM). À partir d'une étude réalisée auprès de 1622 jeunes placés à l'aide sociale à l'enfance (ASE) âgés de 17 à 20 ans, les auteures présentent des parcours différenciés selon que les jeunes s'excluent eux-mêmes de ce dispositif, n'y accèdent pas ou au contraire en bénéficient, dans un contexte de restriction budgétaire particulièrement marqué.

11 Également centré sur une sous-population qui compose la jeunesse, l'article de Yaëlle Amsellem-Mainguy, Benoît Coquard et Arthur Vuattoux interroge le lien entre les trajectoires des mineurs incarcérés et leurs plus ou moins grandes vulnérabilités face à l'expérience de l'incarcération. Les résultats présentés sont issus d'une enquête originale par entretiens auprès des mineurs - garçons et filles - incarcérés et des 
professionnels qui les encadrent. Les auteurs analysent les conséquences des ruptures biographiques, scolaires et amoureuses induites par la ou les incarcérations successives.

À partir des données de l'enquête Trajectoires et Origines (TeO, INED et INSEE, 2008), Jean-Luc Primon et Laure Moguerou prennent pour objet l'impact de la taille de la fratrie sur les trajectoires d'entrée dans la vie active des enfants d'immigrés, en se focalisant sur les premiers pas sur le marché de l'emploi et les effets de la dimension de la famille d'origine et du genre.

La contribution de Khalid Eljim et Mehdi Le Petit Guerin s'intéresse aux jeunes noninsérés et aux jeunes aux RSA, dont les trajectoires relèvent de processus complexes. Cette étude quantitative mêle données de contextualisation et suivi de cohorte afin de déterminer les récentes tendances de l'insertion des jeunes précaires en Gironde.

À partir de l'enquête « Génération » du CEREQ de 2013, Philippe Lemistre propose une analyse de la «démocratisation ségrégative » pour les sortants de l'enseignement supérieur, selon que les parcours d'études concernent des diplômés professionnels ou pas. Il étudie plus particulièrement l'influence de l'origine sociale sur la réussite au diplôme final, la poursuite d'études et l'insertion.

15 À partir d'une diversité d'objets, de terrains, de méthodologies, de perspectives, ce dossier composé de cinq articles entend donc proposer de traiter des vulnérabilités vécues par les jeunes dans leur parcours d'entrée dans la vie adulte et mettre en lumière combien, moins que l'âge, ce sont les dispositions des jeunes qui les exposent davantage à des situations de vulnérabilités. Cette variété de matériaux et de méthodes a enfin pour objectif de souligner l'ouverture et les potentialités que permet l'étude de la question de la jeunesse et des vulnérabilités en termes de parcours et de processus.

\section{BIBLIOGRAPHIE}

Amsellem-Mainguy Y. et Timoteo J. (2012), Atlas des jeunes en France, Paris, Éditions Autrement.

Becquet V. (2012), «Les “jeunes vulnérables" : essai de définition », Agora débats/jeunesses, vol. $62, \mathrm{n}^{\circ} 3$, p. 51-64.

Bourdieu P. (1978), « La jeunesse n'est qu'un mot », in Questions de sociologie, Paris, Minuit.

Brodiez-Dolino A. (2016) « Le concept de vulnérabilité », La Vie des idées [En ligne], 11 février 2016, disponible sur : http://www.laviedesidees.fr/Le-concept-de-vulnerabilite.html.

Castell L., Portela M. et Rivalin R. (2016), « Les principales ressources des 18-24 ans. Premiers résultats de l'enquête nationale sur les ressources des jeunes ", INSEE Première, n 1603.

Cordazzo Ph. et Sembel N. (2016), « Un “désordre” dans la catégorisation : le déclassement statutaire atypique de diplômés du supérieur sans domicile ", Économie et statistiques, n 488-489, p. 69-85. 
Gaubert É., Henrard V., Robert A. et Rouaud P. (2017), « Enquête 2016 auprès de la génération 2013 - Pas d'amélioration de l'insertion professionnelle pour les non-diplômés ", CEREQ - Bref, $n^{\circ} 356$.

Labadie F. (dir.) (2014), « Parcours de jeunes et territoires », rapport de l'observatoire de la jeunesse, Paris, Injep - La documentation française.

Lefresne F. (2003), « II. / Les déterminants de l'accès à l'emploi des jeunes », Les jeunes et l'emploi, Paris, La Découverte, p. 28-47.

Mercat-Bruns M. et Pallares Castany A. (2016), « La faible visibilité juridique de la discrimination dans l'emploi fondée sur le jeune âge ", Agora débats/jeunesses, vol. 74, n³, p. 63-75.

Papon S. et Beaumel C. (2018), « Bilan démographique 2017 », INSEE Première, nº 1683.

Soulet M. (2014), « Justesse, justice et justification. Les embarras de l'action sociale », in Dubet C. (dir.), Inégalités et justices sociale, Paris, La Découverte.

Van de Velde C. (2016), Sociologie des âges de la vie, Paris, Armand Colin.

INDEX

Mots-clés : vulnérabilité, jeunesse, insertion, parcours, trajectoires

Keywords : vulnerability, youth, integration, career paths, trajectories

\section{AUTEURS}

\section{YAËLLE AMSELLEM-MAINGUY}

Chargée de recherche

Institut national de la jeunesse et de l'éducation populaire - INJEP

amsellem-mainguy@injep.fr

\section{PHILIPPE CORDAZZO}

Professeur de démographie

Université de Strasbourg, SAGE-UMR 7363

cordazzo@unistra.fr 